\title{
Performing a task jointly enhances the sound-induced flash illusion
}

\author{
Basil Wahn ${ }^{1}$, Tim Rohe ${ }^{2}$, Anika Gearhart ${ }^{3}$, Alan Kingstone ${ }^{1}$, and Scott Sinnett ${ }^{3}$
}

\begin{abstract}
Our different senses are stimulated continuously. Through multisensory integration, different sensory inputs may or may not be combined into a unitary percept. Simultaneous with this stimulation, people are frequently engaged in social interactions, but how multisensory integration and social processing combine is largely unknown. The present study investigated if, and how, the multisensory sound-induced flash illusion is affected by a social manipulation. In the sound-induced flash illusion, a participant typically receives one visual flash and two auditory beeps and she/he is required to indicate the number of flashes that were perceived. Often, the auditory beeps alter the perception of the flashes such that a participant tends to perceive two flashes instead of one flash. We tested whether performing a flash counting task with a partner (confederate), who was required to indicate the number of presented beeps, would modulate this illusion. We found that the sound-induced flash illusion was perceived significantly more often when the flash counting task was performed with the confederate compared to performing it alone. Yet, we no longer find this effect if visual access between the two individuals is prevented. These findings, combined with previous results, suggest that performing a multisensory task jointly - in this case an audiovisual task - lowers the extent to which an individual attends to visual information - which in turn affects the multisensory integration process.
\end{abstract}

\section{Keywords}

multisensory processing, social cognition, joint action, sound-induced flash illusion, double flash illusion

\section{Introduction}

Humans constantly receive input from multiple sensory modalities. This input may or may not be combined into a unitary percept through the process of multisensory integration (Ernst \& Banks 2002; Ernst \& Bülthoff 2004; Körding et al. 2007; Rohe \& Noppeney 2015). For this process, sensory signals are weighted depending on their reliability. For example, if humans intend to locate an object in space and receive visual and auditory signals from this object, then the more reliable sensory input (usually, the visual signal) is given a higher weight for the integrated percept to attain the best possible estimate of the object's location.

Researchers have investigated a number of factors that may affect the integration process such as, for instance, attentional load (Alsius et al. 2005; Vroomen et al. 2001; Bertelson et al. 2000; Santangelo \& Spence 2008, 2009; Santangelo \& Macaluso 2012; Wahn \& König 2015b,a, 2016; Wahn et al. 2017b), age (Freiherr et al. 2013; Laurienti et al. 2006; Chan et al. 2018), and neurodevelopmental disorders (Chan et al. 2015; Foss-Feig et al. 2010; Stevenson et al. 2014; Williams et al. 2010). However, little research has investigated whether the integration process is also affected by social factors (i.e., whether a task is performed in the presence of, or with, others). This is surprising as in everyday life people often perform crossmodal tasks in the presence of, or with, others. For example, when people share a meal stimuli from several sensory modalities are combined, or when one attends a lecture both visual (e.g., seeing the lecturer) and auditory information (e.g., hearing the lecturer speak) are processed while being in the presence of others.
In addition, a large body of research in the field of joint action has shown that individual task performance is affected by performing visual perceptual tasks jointly (Böckler et al. 2012; Karlinsky et al. 2017; Knoblich \& Sebanz 2006; Sebanz et al. 2003, 2006; Sellaro et al. 2018; Saunders et al. 2018; Vesper et al. 2017; Yamaguchi et al. 2018). For instance, Sellaro et al. (2018) investigated the effects of joint task performance in a visual picture-word interference task. In their study, participants were shown pictures together with either semantically related or unrelated words. Participants performed a picture naming task alone or with a co-actor who was required to perform a word naming task. Sellaro et al. (2018) found that the unrelated words interfered to a lesser extent with the picture naming task when a co-actor was tasked to indicate the presented words, compared to performing the picture naming task alone. To explain these findings, Sellaro et al. (2018) suggested that participants "off-loaded" attending to the words to the co-actor and were therefore less distracted by them. In particular, Sellaro et al. (2018) suggested that participants co-represented the coactor's task, which allowed them to effectively filter out

\footnotetext{
${ }^{1}$ Department of Psychology, University of British Columbia, Vancouver, Canada

2 Department of Psychiatry and Psychotherapy, University of Tübingen, Tübingen, Germany

${ }^{3}$ Department of Psychology, University of Hawai'i at Mānoa, Honolulu, USA

Corresponding author:

Basil Wahn, Department of Psychology, University of British Columbia, 2136 West Mall, V6T 1Z4, Vancouver, Canada

Email: bwahn@uos.de
} 
the co-actor's task from their own task representation. The process of co-representation (i.e., that co-actors take into account each other's tasks) has been proposed to occur automatically when humans perform tasks together (Sebanz et al. 2003) and it has been suggested to form the basis for more complex joint actions (Vesper et al. 2010).

The first study (Heed et al. 2010) that investigated how joint task performance influences multisensory processing (as opposed to only visual processing) considered visuotactile integration in a tactile localization task. Heed et al. (2010) required participants to localize tactile stimuli while visual stimuli were presented from either the same spatial location as the tactile stimuli (congruent presentations) or from a different location (incongruent presentations). The standard finding in a task of this type is that although participants integrate the visual and tactile stimuli even when they are presented from different spatial locations, tactile location judgments are negatively affected by the visual stimuli for incongruent presentations. For example, participants tend to be slower to localize the tactile stimuli for incongruent compared to congruent presentations - an effect referred to as a "crossmodal congruency effect" (Spence et al. 2004). When participants performed this task alone, Heed et al. (2010) replicated this crossmodal congruency effect (Spence et al. 2004), finding that participants were slower to localize the tactile stimuli for incongruent compared to congruent presentations.

To test whether social factors affect multisensory integration, participants in Heed et al. (2010)'s experiment also performed the tactile localization task in the presence of another person, or with a co-actor who was required to localize the visual stimuli. Their results demonstrated that the crossmodal congruency effect was reduced when participants performed the task with a co-actor, suggesting that visuotactle integration is affected by performing a task jointly. Heed et al. (2010) posited that these findings suggest a re-weighting of the reliabilities for the different sensory modalities. In particular, they proposed that having a co-actor respond to the visual stimuli reduced the weighting of the visual stimuli for visuotactile integration. As in the study discussed above (Sellaro et al. 2018), Heed et al. (2010) also suggest that the mechanism of task co-representation has allowed co-actors to filter out the co-actor's task representation from their own task representation. Hence, co-representing the co-actor's task may have allowed participants to filter out the presented visual information while performing the tactile localization task. As a result, the visual stimuli distracted to a lesser extent from the task of localizing tactile stimuli (i.e., participants offload the visual information to the co-actor), leading to a reduction of the crossmodal congruency effect. In other words, offloading stimuli to a co-actor may have resulted in a stronger segregation of sensory streams, which in turn reduced the effect of the distracting visual stimuli. As a point of note, Heed et al. (2010) only analyzed performance in the tactile localization task as the tactile stimuli did not affect performance in the visual localization task.

While the findings by Heed et al. (2010) suggest that visuotactile integration is susceptible to social factors, we tested the robustness of this effect in an analogous crossmodal congruency task involving audiovisual spatial stimuli instead of visuotactile spatial stimuli (Wahn et al. 2017a). Participants received auditory and visual stimuli that were either spatially congruent or incongruent, and were required to perform an auditory localization task either alone or with a co-actor, whose task was to localize the visual stimuli. Convergent with Heed et al. (2010), we found that the crossmodal congruency effect, measured again in response time, was modulated when the task was performed jointly, suggesting that social factors not only affect visuotactile integration but also audiovisual integration. Again, the reasoning used to explain these effects is that the reliability of the stimuli, to which the co-actors respond, is reduced, as participants can filter out the co-actor's task representation from their own task representation. As a result, participants' location judgements of the auditory stimuli are influenced to a lesser degree by incongruent visual stimuli. Analogously to Heed et al. (2010), Wahn et al. (2017a) only analyzed performance in the auditory localization task as the auditory stimuli did not affect performance in the visual localization task.

In a recent study (Wahn et al. in press), we aimed to further extend these findings to other crossmodal paradigms to test to what extent earlier effects generalize to other crossmodal phenomena. In particular, we tested whether joint task performance affects audiovisual integration in a motion discrimination task (Soto-Faraco et al. 2002) and a temporal order judgment task (Morein-Zamir et al. 2003). In this study, we again used a similar design as in earlier studies (Heed et al. 2010; Wahn et al. 2017a). In particular, one participant responded to stimuli in the visual sensory modality and one in the auditory sensory modality. While we replicated earlier crossmodal effects, we did not find that audiovisual integration was affected by joint task performance. These findings suggest that the effect of joint task performance on multisensory integration is highly taskdependent.

Given that the research described above shows that performing a task jointly does not influence multisensory integration in all crossmodal tasks, it is important to explore further which crossmodal phenomena are susceptible to social factors. To date, it is unknown whether nonspatial crossmodal illusions are influenced by joint task performance. In other words, it is unknown whether the suggested underlying mechanism of co-representation is specific only to spatial stimuli or also extends to nonspatial crossmodal illusions. A robust nonspatial crossmodal illusion that has been investigated extensively is the soundinduced flash illusion (also referred to as the "fission illusion") (Cuppini et al. 2014; Mishra et al. 2007, 2010; Shams et al. 2002, 2005b,a; Shams \& Kim 2010). In a typical experiment investigating this illusion, participants see a variable number of flashes on a computer screen while also hearing a variable number of auditory beeps presented in a rapid temporal sequence. When participants are presented with a single flash and two (or more) beeps, they tend to perceive more than one flash despite only one flash having been objectively presented. In other words, the flash is "split in two" by the two auditory beeps, hence the name "fission illusion". The reasoning behind this illusion is that humans tend to give a considerably higher reliability to the auditory signal compared to the visual signal in tasks that involve 
temporal precision (Shams et al. 2002). As a result, the auditory signal may dominate the integrated percept, leading to the perception that two flashes occurred.

Another related illusion that has been investigated, although to a lesser extent, is the "fusion illusion" (Andersen et al. 2004; Vanes et al. 2016). In this illusion, when participants receive two flashes and one beep, participants may perceive one flash instead of two flashes. That is, the two flashes tend to be "fused" into one. As with the fission illusion, the explanation for the fusion illusion is that the auditory signal dominates the integrated percept, altering the perceived number of flashes. Yet, it also has been suggested that the underlying mechanism for this type of illusion differs from the fission illusion. In particular, researchers found that the fission illusion is affected by age while this is not the case for the fusion illusion (McGovern et al. 2014; Parker \& Robinson 2018), occipital stimulation interferes with fission but not fusion effects (Bolognini et al. 2011), and different ERP components appear to contribute towards the fusion and fission illusions (Mishra et al. 2008). Finally, fission illusion effects tend to be consistently larger than fusion illusion effects across studies (Bolognini et al. 2011; McGovern et al. 2014; Mishra et al. 2008). Given that these two types of illusions are likely mediated via different mechanisms, it is hence important to investigate whether both illusions are influenced by social factors or not.

Taken together, it is not known whether nonspatial crossmodal illusions are susceptible to social factors or not. Yet, given that the effect of social factors on multisensory integration appears to be highly task-dependent (Wahn et al. in press), it is important to test whether earlier findings (Heed et al. 2010; Wahn et al. 2017a) generalize to nonspatial crossmodal illusions or not. That is, it is unknown whether the mechanism of task co-representation also influences nonspatial crossmodal illusions or is unique to crossmodal spatial localization tasks. In the present study, we address this gap in the literature by testing whether the fission and fusion illusions are affected by the presence of another person and/or a co-actor with whom the task is performed jointly. In particular, following the same experimental design logic as in the studies discussed above (Heed et al. 2010; Wahn et al. 2017a, in press), a participant is instructed to perform a task in the sensory modality in which crossmodal influences are known to occur (here, a visual flash counting task) either alone, in the presence of another person, or with a co-actor who will be required to respond to events in a different sensory modality (here, an auditory beep counting task). If the reliability of the sensory modality to which a coactor responds is affected (as it has been suggested in earlier studies Heed et al. (2010); Wahn et al. (2017a)), then we would expect a reduction of the perception of the illusion when the task is performed jointly. That is, the influence of the auditory information should have a reduced influence on the perception of the flashes. Yet, another possibility is that the reliability of the visual sensory modality is affected by performing a task jointly regardless of which co-actor is in charge to respond to visual events. In this case, the perception of the illusion would be increased as the auditory information would have a higher influence on the perceived number of visual flashes.

\section{Materials and Methods}

\section{Participants}

36 students ( 18 female, $M=19.31$ years, $S D=2.51$ years) of the University of Hawai'i at Mānoa participated in our main experiment and 34 students (19 female, $M=18.91$ years, $S D=1.29$ years) participated in an additional control experiment. The study was approved by the Committee on Human Studies of the University of Hawai'i at Mānoa. Participants received course credits as a compensation for their participation. All participants had normal or correctedto-normal vision and hearing. A confederate (female, 22 years old) for the purpose of our social manipulations participated in the experiment. The confederate was also a student at the University of Hawai'i at Mānoa, but was not known to the participants.

To assess the statistical power in the present study (O'Keefe 2007), we ran a sensitivity analysis (alpha $=0.05$, power $=0.80$ ) for a two-sided paired t-test using the software $\mathrm{G}^{*}$ Power and found that we have sufficient power to detect moderately sized effects both for our main (Cohen's $\mathrm{d}=$ 0.48) and control experiment (Cohen's $d=0.50$ ), which is sufficient as the effect size found in our earlier study (Wahn et al. 2017a) was larger (Cohen's d =0.93).

\section{Experimental setup}

The participant and the confederate sat next to each other in front of a computer screen (iMac, 21.5-inch, mid 2011 model; resolution: 1920 x 1080; refresh rate: $60 \mathrm{~Hz}$ ). Their heads were aligned to the outer edges of the screen (i.e., if they look straight they see the outer edges of the screen) at a distance of approximately $60 \mathrm{~cm}$. Auditory stimuli (beeps) $(450 \mathrm{~Hz}$; duration: $5 \mathrm{~ms}, \mathrm{SPL}=8.5 \mathrm{dBA})$ were played on speakers placed next to the computer screen (see Figure 1, for an overview). Responses were made on separate keyboards that were placed in front of each of them. The seating positions of the participant and confederate were counterbalanced across participants (i.e., for half of the measurements, the participant was sitting on the right and the confederate on the left, for the other half of the measurements the seating was reversed). For the control experiment, we additionally placed a divider between participants such that the confederate and participants no longer could see each other but could clearly view the stimuli on the computer screen.

\section{Experimental procedure}

For each trial, the participant's primary task was to indicate whether one or two flashes were shown ("flash counting task") using her/his assigned keys. If the participant was sitting on the right, she/he would be required to press the " $\mathrm{K}$ " key to indicate one flash or the adjacent "L" key to indicate two flashes. If the participant was sitting on the left, the participant would be required to press the " $\mathrm{S}$ " key for one flash or the adjacent " $D$ " key for two flashes. The flashes (2.52 visual degrees wide) were presented 6.29 visual degrees below a fixation dot (0.56 visual degrees radius). One flash or two flashes were presented either together with one or two beeps (all stimuli combinations and the temporal profile of how stimuli were presented can be viewed in 


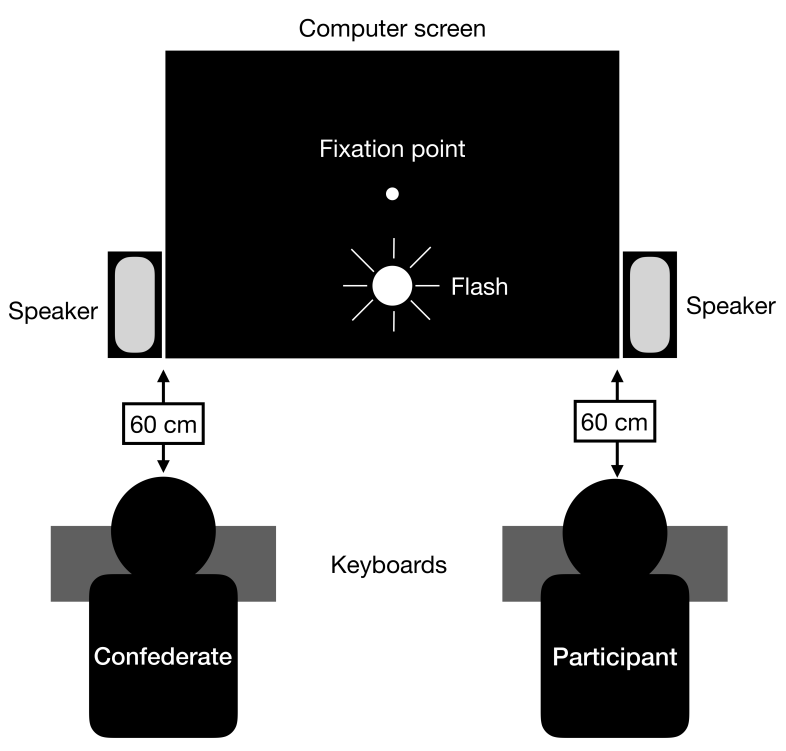

Figure 1. Experimental setup overview.

Figure 2). While performing this task, the participant was required to fixate the fixation dot in the center of the screen. The combination of number of flashes and beeps were randomly selected for each trial. The fission illusion was expected to occur when participants received one flash and two beeps. The fusion illusion was expected to occur when participants received two flashes and one beep. No illusions were expected for all other combinations of flashes and beeps, i.e., where the number of flashes and beeps were the same (i.e., when either 2 flashes and 2 beeps or 1 flash and 1 beep were presented).

As a point of note, in the original study by Shams et al. (2002) the fission illusion was perceived almost on every trial. Exactly replicating the original study would have hence produced an illusion, for which we could not assess whether it is enhanced due to our social manipulations or not. Hence, we chose to present the flashes longer in the present study (33 ms) when compared with the original study by Shams et al. (2002) (17 ms) to increase the weight of the visual sensory modality for the multisensory integration process and thereby reduce the occurrence of the fission illusion.

To ensure that participants maintained fixation at the central fixation dot, on $20 \%$ of randomly selected trials, a flash (0.70 visual degrees radius) would briefly replace the central fixation dot for $50 \mathrm{~ms}$. In these trials, no flashes and beeps were presented and participants were required to press a different key than those required for the flash counting task ("enter" if the participant is sitting on the right or "space" if the participant is sitting on the left). This task will be referred to as the "fixation control task".

The participant performed these two tasks (i.e., the flash counting task and fixation control task) throughout the whole experiment while we manipulated the social factor in three different conditions ("individual", "joint passive", and "joint active") in a within-subject design. In the individual condition, the participants performed the two tasks alone. For this condition, the confederate was asked to wait outside of the experimental room. A trial was completed for the individual condition, when the participant, who was still

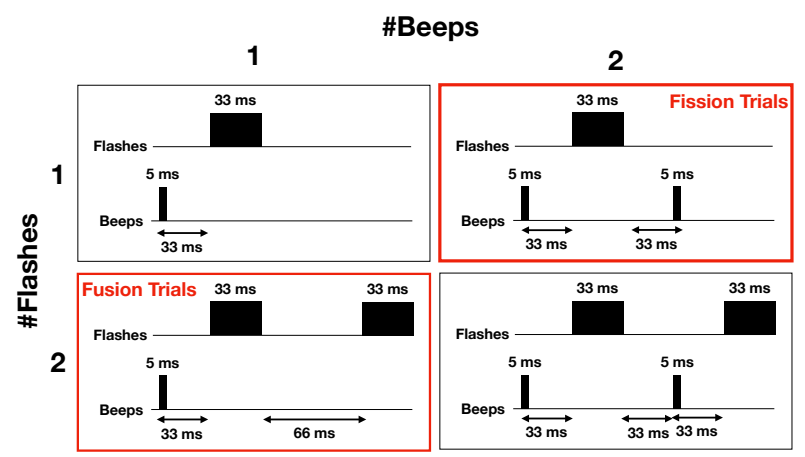

Figure 2. Stimuli combinations and temporal order of stimuli presentations. For each combination of stimuli (i.e., 1 Flash \& 1 Beep (upper left); 1 Flash \& 2 Beeps (upper right); 1 Beep \& 2 Flashes (lower left); 2 Beeps \& 2 Flashes (lower right), the order in which stimuli were presented is depicted. Trials in which an illusion may occur (i.e., "Fission" and "Fusion" trials) are framed in red.

positioned on the left or right, made a response pressing either one of the keys to indicate the number of flashes or the key to indicate a flash in the center. Note, any of these key responses would end a trial regardless of the task (i.e., flash counting task or fixation control task).

In the joint passive condition, the confederate would sit next to the participant and was instructed to perform the fixation control task as well as the participant. That is, whenever there was a flash in the center, the confederate was also required to respond with a key ("enter" if the confederate was sitting on the right; "space" if the confederate was sitting on the left).

In the joint active condition, the confederate would be required to indicate the number of perceived beeps using her/his assigned keyboard in addition to performing the fixation control task. In this condition, a trial ends after both the participant and confederate had made responses (i.e., pressed any of their assigned keys).

Note, in all experimental conditions, the task requirements remained the same for the participants (i.e., they performed the flash counting task and the fixation control task). Only the confederate's task requirements changed depending on the experimental conditions. The participant was aware of the confederate's task requirements in all conditions as the participant was also present when the experimenter explained the confederate's task to the confederate. Moreover, at the beginning of each joint block, the experimenter reminded the confederate of their assigned tasks. The seating positions of the participant and the confederate remained the same regardless of the experimental conditions throughout the experiment. Only across participants, the seating positions of the confederate and the participant were counterbalanced.

The three conditions were performed in three blocks of 150 trials each. Within each block, 30 trials for the fixation control task and 30 trials for each combination of flashes and beeps (i.e., 1 Beep / 1 Flash, 1 Beep / 2 Flashes, 2 Beeps / 1 Flash, 2 Beeps / 2 Flashes) were performed in a randomized sequence. The program automatically continued to the next trial after the required responses were made and there was a $1000 \mathrm{~ms}$ break between trials. Before the 
start of each block, participants saw text on the computer screen announcing the block type and requesting that the experimenter be contacted. The experimenter then made the necessary setup adjustments depending on the block type (e.g., asking the confederate to wait outside for the individual condition block). For each participant, the order of experimental blocks was randomly selected. The experiment took about 20 minutes to complete. After the experiment was completed, participants were debriefed about the purpose of the experiment and were notified that the co-actor was a confederate. The experiment was programmed using python (version 2.7) and the pygame library.

\section{Methods of data analysis}

As the assumption of normality was violated for all our tests (repeated measures ANOVA and t-test), we use permutationbased repeated measures ANOVAs and permutation-based paired t-tests. That is, the sampling distribution under the null hypothesis was estimated by repeatedly sampling permutations of the data (Kherad-Pajouh \& Renaud 2015). For the pairwise comparisons, we corrected p-values for multiple comparisons using the Bonferroni correction and report corrected $\mathrm{p}$ values. For the ANOVAs, we report $\eta^{2}$ as an effect size measure and for all t-tests we report Cohen's d. All analyses were performed in R (R Core Team 2017) using custom scripts.

Prior to our analysis, we also assessed whether all participants were performing the fixation control task accurately and calculated the proportion of correct responses for the fixation control task trials for each condition. We found that participants performed the fixation control task with a high accuracy for all conditions in our main experiment (individual: $M=0.97, S D=0.06$; joint passive: $M=0.96, S D=0.06$; joint active: $M=0.97, S D=0.04$ ) and control experiment (individual: $M=0.98, S D=0.03$; joint passive: $M=0.98, S D=0.03$; joint active: $M=0.97$ $S D=0.04)$.

\section{Results}

\section{Fission illusion}

To examine whether participants perceived the fission illusion and how the strength of this illusion may be altered depending on the manipulated social factors, we plotted the average responses for the presentations involving one flash with either one or two beeps (i.e., the conditions leading to the fission illusion), separately for the three conditions (individual, joint passive, and joint active) in Figure 3A (left panel). On a descriptive level, one sees that participants tend to perceive the fission illusion and that the strength of this illusion is influenced our social manipulation. That is, the illusion appears to be strongest for the joint active condition followed by the joint passive and individual condition.

To test if these observations were statistically significant, we performed a permutation-based $2 \times 3$ repeated measures ANOVA with the factors Beep Number $(1,2)$ and Condition (individual, joint passive, joint active) when only one flash was presented (top row in Figure 2). We found a significant main effect of Beep Number $(F(1,35)=56.94, p<$ $\left..001, \eta^{2}=.272\right)$, suggesting that participants perceived the
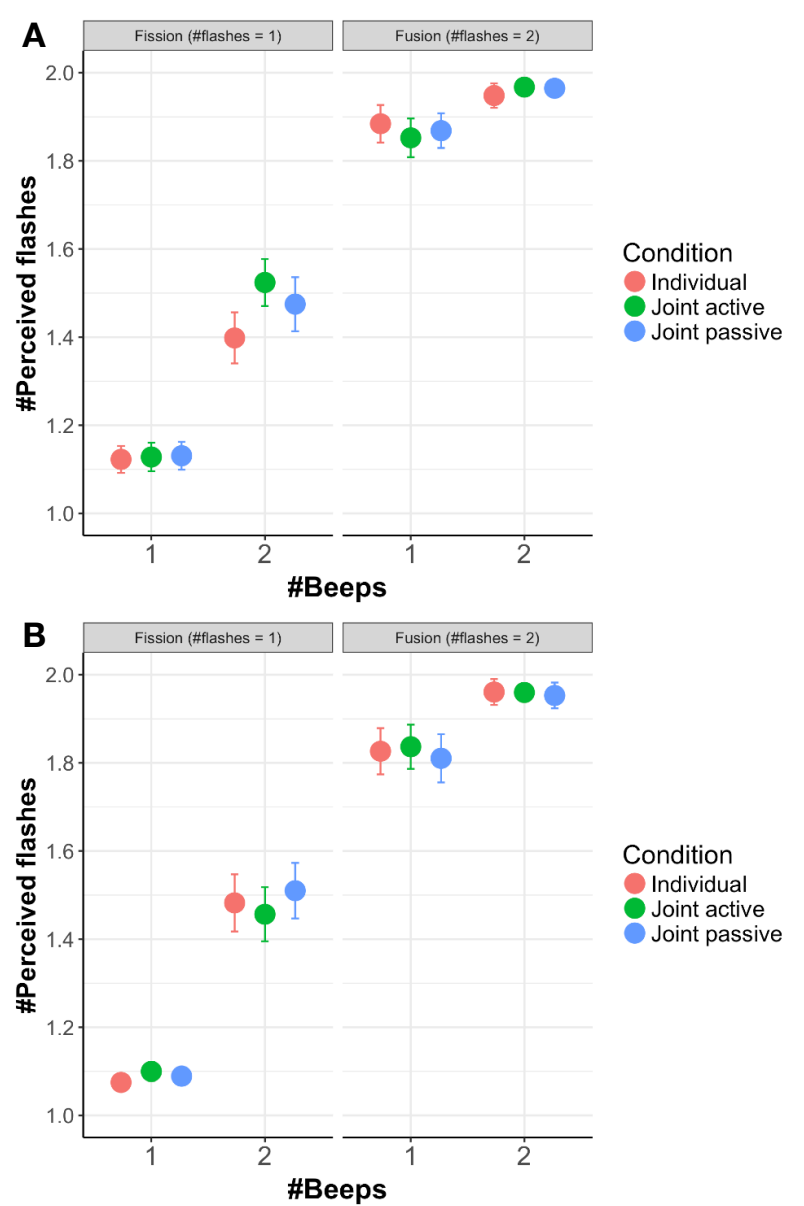

Figure 3. Results overview for the fission (left panel) and fusion (right panel) illusion. Averaged responses of perceived number of flashes as a function of beep number, for the individual, joint passive, and joint active conditions for trials in which either one flash was shown (left panel) or two flashes were shown (right panel). In A, data from our main experiment are shown. In $\mathrm{B}$, data from our control experiment are shown. Error bars are standard error of the mean.

illusion, and a significant main effect of Condition $(F(2,70)$ $\left.=3.97, p=.020, \eta^{2}=.007\right)$. Importantly, we found a significant interaction effect $\left(F(2,70)=5.22, p=.006, \eta^{2}=\right.$ .006), suggesting that the fission illusion was affected by our social manipulations.

We followed up this interaction effect with pairwise comparisons to test how the illusion was influenced by our social manipulations. We first calculated the difference in responses between one beep and two beeps for each condition and then conducted pairwise comparisons between these computed differences using permutation-based paired t-tests. We found significant differences between the joint active condition and the individual condition $(t(35)=-3.50$, corrected $p=.006$, Cohen's $d=-0.41)$, while we did not find significant differences when comparing the individual with the joint passive condition $(t(35)=-2.06$, corrected $p=.138$ Cohen's $d=-0.23)$ or the two joint conditions $(t(35)=1.19$, corrected $p=.780$, Cohen's $d=0.16$ ). In sum, our results suggest that participants perceived the fission illusion and that this illusion was significantly larger in the joint active condition compared to the individual condition. 
To have a performance measure which accounts for potential response biases, we also calculated d' (Green \& Dai 1991; Green et al. 1966) and repeated the analysis above with d' as our dependent variable (for a descriptive overview, see Figure 4A left panel). For the 2x3 ANOVA, we again found a significant main effect of Beep Number $\left(F(1,35)=59.65, p<.001, \eta^{2}=.259\right)$ and, importantly, a significant interaction effect $\left(F(70)=7.65, p=.001, \eta^{2}=\right.$ $.009)$. The main effect of Condition was no longer significant $\left(F(2,70)=2.82, p=.066, \eta^{2}=.005\right)$. Following up the interaction effect with pairwise comparisons, we found significant differences between the joint active condition and the individual condition $(t(35)=4.05$, corrected $p=.006$, Cohen's $d=0.49$ ) and also when comparing the individual with the joint passive condition $(t(35)=2.89$, corrected $p=.006$, Cohen's $d=0.37$ ). The two joint conditions again did not significantly differ $(t(35)=-0.92$, corrected $p=1.00$, Cohen's $d=-0.13)$. In sum, also for d' we find that participants perceived the fission illusion and that the fission illusion is significantly larger in the joint active condition. In addition, we also find that the fission illusion is also enhanced in the joint passive condition relative to the individual condition.

Overall, our findings suggest that we replicate the fission illusion and that this illusion is enhanced in the joint active condition for both our dependent variables. For d', we also find that the illusion is enhanced in joint passive condition relative to the individual condition.

In a control experiment, we tested whether the observed social effects are dependent on the visibility of the co-actor by placing a divider between the participant and confederate. Apart from this manipulation, the experiment remained the same as before. We performed the same permutation-based $2 \times 3$ repeated measures ANOVA as above for each of our dependent variables (i.e., the perceived number of flashes and d'). For the perceived number of flashes variable (for a descriptive overview, see Figure 3B left panel), we again find a main effect of Beep Number $(F(1,33)=55.00, p$ $\left.<.001, \eta^{2}=.368\right)$ but no longer a significant main effect of Condition $\left(F(2,66)=0.52, p=.600, \eta^{2}=.001\right)$ or interaction effect $\left(F(2,66)=1.44, p=.236, \eta^{2}=.002\right)$. For d' (for a descriptive overview, see Figure 4B left panel), we found the same pattern of results: A significant main effect of Beep Number $\left(F(1,33)=61.30, p<.001, \eta^{2}=.360\right)$ but no significant main effect of Condition $(F(2,66)=0.77, p=$ $\left..462, \eta^{2}=.001\right)$ or no significant interaction effect $(F(2,66)$ $\left.=0.94, p=.406, \eta^{2}=.001\right)$.

In addition, we also directly compared the difference in the fission illusion size between the joint active and individual condition across experiments using a two sample t-tes. We found significant differences when using the perceived number of flashes $(t(68)=3.06, p=.003$, Cohen's $d=$ $0.73)$ and d' $(t 68)=3.25, p=.002$, Cohen's $d=0.78)$ as the dependent variable.

Given that we find a difference between the individual condition and joint active condition only for our main experiment but not for our control experiment, one potential explanation for this difference is that response times between the participant and confederate systematically differed for the main experiment but not for the control experiment. For the main experiment, the response times between the
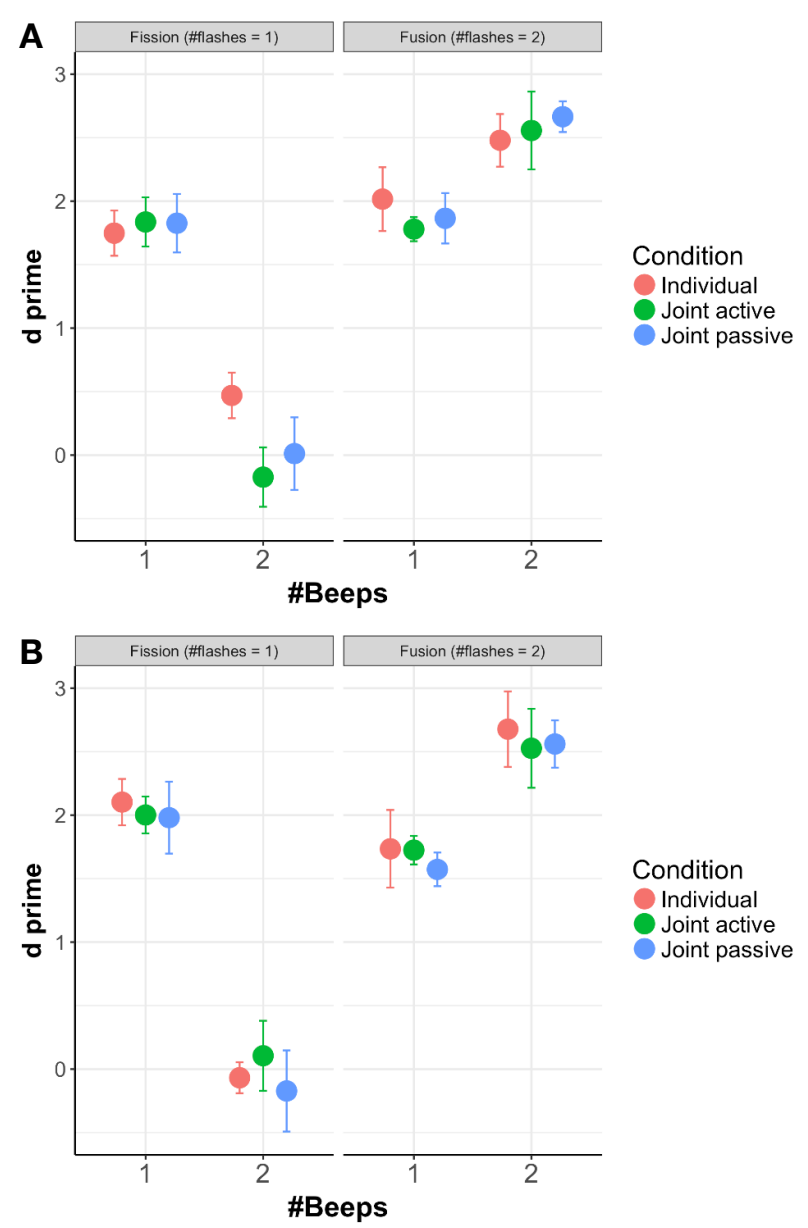

Figure 4. Results overview for the fission (left panel) and fusion (right panel) illusion. Averaged d' as a function of beep number, for the individual, joint passive, and joint active conditions for trials in which either one flash was shown (left panel) or two flashes were shown (right panel). In A, data from our main experiment are shown. In B, data from our control experiment are shown. Error bars are standard error of the mean.

participants $(M=0.73$ seconds, $S D=0.27$ seconds $)$ and confederate $(M=0.61$ seconds, $S D=0.34$ seconds) significantly differed by about $115 \mathrm{~ms}(t(35)=3.14, p=$ .003 , Cohen's $d=0.35)$. For the control experiment, the response times between the participants $(M=0.72$ seconds, $S D=0.15$ seconds $)$ and confederate $(M=0.64$ seconds, $S D=0.14$ seconds) significantly differed by about $80 \mathrm{~ms}$ $(t(33)=2.96, p=.006$, Cohen's $d=0.55)$. When comparing these differences with each other, we found no significant difference $(t(68)=0.77, p=.442$, Cohen's $d=0.18)$. In sum, we found a difference in response times between the confederate and participants for both experiments. Moreover, the size of this response time difference is comparable between the main and control experiment (i.e., only differs by about $35 \mathrm{~ms}$ on average). Hence, our social effects in the main experiment are not explained by systematic differences in response times.

\section{Fusion illusion}

We also tested whether the fusion illusion is influenced by our social manipulations. As a reminder, in the fusion 
illusion participants are presented with two flashes and one beep, with the hypothesis that they will perceive one flash instead of two as the auditory stimuli dominate the integrated percept (Andersen et al. 2004; Vanes et al. 2016). On a descriptive level (see Figure 3A right panel), one sees that participants tend to perceive the fusion illusion (but to a lesser extent than the fission illusion) and that the strength of the illusion is slightly increased for the joint active and passive condition compared to the individual condition. We performed a permutation-based $2 \times 3$ repeated measures ANOVA with the factors Beep Number $(1,2)$ and Condition (Individual, Joint passive, Joint active) for the data for which two flashes were presented (Figure 2). We found a significant main effect of Beep Number $(F(1,35)=6.53, p$ $\left.=.013, \eta^{2}=.052\right)$, suggesting that participants perceived the fusion illusion. Yet, we did not find a significant main effect of Condition $\left(F(2,70)=0.04, p=.962, \eta^{2}=.001\right)$ or interaction effect $\left(F(2,70)=1.97, p=.151, \eta^{2}=.003\right)$. As above, to have a measure which accounts for potential response biases, we again computed d' and repeated the same ANOVA with d' as the dependent variable (see Figure $4 \mathrm{~A}$ right panel, for a descriptive overview). We generally found the same pattern of results: A significant main effect of Beep Number $\left(F(1,35)=12.83, p=.001, \eta^{2}=.087\right)$ but no significant main effect of Condition $(F(2,70)=0.19$, $\left.p=.828, \eta^{2}=.001\right)$ and no significant interaction effect $\left(F(2,70)=2.46, p=.087, \eta^{2}=.004\right)$.

Overall, these results suggest that participants also perceive the fusion illusion. However, when comparing the magnitude of the fusion illusion with the magnitude of the fission illusion (i.e., the difference in perceived flashes between conditions when one or two beeps were presented), we found that the magnitude of the fusion illusion was significantly smaller than for the fission illusion $(t(35)=-$ 5.67, $p<.001$, Cohen's $d=-0.82)$. These results were also found when using d' as a dependent measure $(t(35)=$ $4.30, p<.001$, Cohen's $d=1.32$ ). Importantly with regard to our research question whether social factors affect nonspatial crossmodal illusions, our results suggest that our social manipulations did not affect the fusion illusion.

We repeated the analysis above also for our control experiment, in which co-actors could no longer see each other. For the ANOVA using the indicated number of flashes as dependent variable (for a descriptive overview, see Figure 3B right panel), we again replicated the fusion illusion, finding a main effect of Beep Number $(F(1,33)=8.22, p=$ $\left..005, \eta^{2}=.089\right)$. The main effect of Condition $(F(2,66)=$ $\left.0.20, p=.832, \eta^{2}=.001\right)$ and the interaction effect $(F(2,66)$ $=0.45, p=.636, \eta^{2}=0$ ) were not significant. For d' (for a descriptive overview, see Figure $4 \mathrm{~B}$ right panel), we find the same pattern of results: A significant main effect of Beep Number $\left(F(1,33)=14.07, p<.001, \eta^{2}=.104\right)$ but no significant main effect of Condition $(F(2,66)=0.360, p=$ $\left..707, \eta^{2}=.002\right)$ or interaction effect $(F(2,66)=0.417, p=$ $\left..421, \eta^{2}=.001\right)$.

\section{Discussion}

The present study investigated if social factors modulate the fission and fusion illusion. Participants performed a flash counting task either alone, in the presence of a confederate, or jointly with a confederate. We replicated the fission illusion, finding that participants tend to perceive two flashes when only one flash and two beeps are presented. Importantly, we also find that, relative to performing the flash counting task alone, this fission illusion is enhanced when the flash counting task is performed with an active coactor whose task is to indicate the number of beeps. When accounting for potential response biases by using d' as a dependent measure, we also find that the fission illusion is enhanced when the flash counting task is performed in the presence of a passive co-actor. These results suggest that the sound-induced flash illusion is affected by social factors.

For the fusion illusion, we find that participants also tend to perceive this illusion but to a considerably lesser degree than the fission illusion. Moreover, our social manipulations did not affect the strength of the fusion illusion. As noted in the introduction, prior research suggests that fission and fusion illusion are mediated by different mechanisms (Mishra et al. 2008; McGovern et al. 2014; Parker \& Robinson 2018). For example, the fission illusion is affected by age (McGovern et al. 2014; Parker \& Robinson 2018) and occipital stimulation (Bolognini et al. 2011) while that is not the case for the fusion illusion, suggesting that the fission illusion may be more malleable than the fusion illusion. Our findings dovetail with this proposition, as social factors only affected the fission illusion. In other words, unlike the fission illusion, the fusion illusion may be mediated by a mechanism that is insensitive to social manipulations. Nevertheless, it is also the case that the illusions differed in strength (fission $>$ fusion), and so it is possible that a sufficiently large crossmodal effect may be a prerequisite to observing significant social effects. A future study could test whether social effects would be present with a greater baseline fusion illusion.

When considering previous studies that investigated the influence of social factors on crossmodal spatial congruency tasks (Heed et al. 2010; Wahn et al. 2017a), one important point to note is that performing a task jointly led to a reduction in distraction for incongruent visual stimuli. In particular, when investigating a spatial visuotactile congruency effect, Heed et al. (2010) found that participants performing a tactile localization task were less distracted by incongruent visual stimuli when a coactor was required to indicate where the visual stimuli were located. Similarly, in our previous study involving a spatial audiovisual congruency task (Wahn et al. 2017a), participants performing an auditory localization task were less distracted by incongruent visual stimuli when a co-actor was required to indicate the location of the visual stimuli. In the present study, however, we found that the crossmodal illusion was enhanced when participants indicated the number of visual flashes and a co-actor was required to indicate the number of auditory beeps. At first blush, this may appear to be at odds with previous findings given that we found an enhanced crossmodal illusion in the current study, and earlier studies recorded a reduction in the crossmodal congruency effect. Yet, these findings can be reconciled by the proposal that performing a task jointly specifically affects the reliability of stimuli in the visual sensory modality rather than the sensory modality for which a co-actor is required to make responses. In particular, we suggest that visual 
attention was divided between the visual flash counting task and attending the co-actor during joint task performance. This had the effect that participants attended to a lesser extent to the visual stimuli, which they were required to count. As a result, the reliability of the visual stimuli was reduced and the auditory stimuli dominated the integrated percept more than before, increasing the perception of the fission illusion. On a related note, dual task crossmodal research has shown that using a secondary task to divert attention from a primary task can reduce the reliability of the sensory modality in which the primary task is performed (Helbig \& Ernst 2008; Wahn \& König 2016). As a result, stimuli from the sensory modality of the primary task are weighted less for the multisensory integration process. Similarly, we propose that participants in the present study were distracted by the co-actor, which divided their visual attention. Supporting this proposal, when a divider prevents co-actors from seeing one another, and therefore prevents the allocation of visual attention to their co-actor, the social enhancement of the fission illusion is abolished.

Alternatively, one could suggest that these effects are (at least in part) explained by social impairment effects (for a recent review, see Belletier et al. (2019)). That is, simply the presence of another person may already produce a reduced performance on the visual counting task. Supporting this proposal, we also find that the fission illusion was significantly enhanced in the presence of a passive confederate relative to the individual condition - at least, when using d' as the dependent measure. Yet, we only found a significant difference for both our dependent variables (i.e., the perceived number of flashes and d') between the individual condition and the joint active condition. At present, our findings suggest that social presence effects may only in part drive present social effects and that having an active confederate is critical.

Relatedly, the present findings are unlikely to be explained by the idea that participants were less willing to attend to the visual stimuli (i.e., reduced task compliance) in the joint conditions, as we did not find any social effects for the fusion illusion and the control experiment. Future studies could extend the present findings by investigating whether participation of an active co-actor affects other crossmodal phenomena and whether potential social effects depend on visual access to the co-actor. Taking a different tack, future studies could also test how other social manipulations could affect multisensory integration as, for instance, an ingroupoutgroup manipulation of the confederate, as this has been found to alter social processing in other joint action studies (Iani et al. 2011; Müller et al. 2011; McClung et al. 2013).

On a more general level, we also want to propose a reason why social manipulations could affect multisensory processing in the first place given that prior research has largely been silent on this issue. Besides constantly processing multisensory stimuli in daily life, it is social experiences that shape human life. And naturally, these two types of experiences often coincide. Moreover, seminal work in the field of social cognition has highlighted that the social environment humans live in has fundamentally affected the formation of their neurocognitive system. In particular, researchers have proposed that the need to cope with increased social processing demands may be why the human brain evolved to its relatively large size and complexity (Frith 2007). Also, there is evidence suggesting that several brain structures originally involved in temporal and spatial perceptual processing have been repurposed for social processing (Parkinson \& Wheatley 2015). Hence, brain areas involved in perceptual processing are (at least in part) also involved in social processing. We speculate that this overlap in neural processing could help explain the effects of joint task performance on multisensory processing, as similar brain areas may be involved in social and multisensory processing. Future neurophysiological studies could test this provocative proposal by investigating the extent of overlap between brain regions involved in social and multisensory processing.

\section{Funding}

We acknowledge the support of a DFG research fellowship (WA 4153/2-1) awarded to BW, and a NSERC Discovery grant and SSHRC Insight grant awarded to AK.

\section{Declaration of conflicting interests}

The authors declare no conflicting interests.

\section{References}

Alsius A, Navarra J, Campbell R, Soto-Faraco S, 2005 "Audiovisual integration of speech falters under high attention demands" Current Biology 15 839-843

Andersen T S, Tiippana K, Sams M, 2004 "Factors influencing audiovisual fission and fusion illusions" Cognitive Brain Research 21 301-308

Belletier C, Normand A, Huguet P, 2019 "Social-facilitation-andimpairment effects: From motivation to cognition and the social brain" Current Directions in Psychological Science 28260 265

Bertelson P, Vroomen J, De Gelder B, Driver J, 2000 "The ventriloquist effect does not depend on the direction of deliberate visual attention" Perception \& Psychophysics 62 321-332

Böckler A, Knoblich G, Sebanz N, 2012 "Effects of a coactor's focus of attention on task performance." Journal of Experimental Psychology: Human Perception and Performance 381404

Bolognini N, Rossetti A, Casati C, Mancini F, Vallar G, 2011 "Neuromodulation of multisensory perception: a tdcs study of the sound-induced flash illusion" Neuropsychologia 49 231237

Chan J, Kaiser J, Brandl M, Matura S, Prvulovic D, J Hogan M, J Naumer M, 2015 "Expanded temporal binding windows in people with mild cognitive impairment" Current Alzheimer Research 12 61-68

Chan J S, Connolly S K, Setti A, 2018 "The number of stimulusonset asynchronies affects the perception of the sound-induced flash illusion in young and older adults" Multisensory Research 31 175-190

Cuppini C, Magosso E, Bolognini N, Vallar G, Ursino M, 2014 "A neurocomputational analysis of the sound-induced flash illusion" Neuroimage 92 248-266

Ernst M O, Banks M S, 2002 "Humans integrate visual and haptic information in a statistically optimal fashion" Nature 415 429433 
Ernst M O, Bülthoff H H, 2004 "Merging the senses into a robust percept" Trends in Cognitive Sciences 8 162-169

Foss-Feig J H, Kwakye L D, Cascio C J, Burnette C P, Kadivar H, Stone W L, Wallace M T, 2010 "An extended multisensory temporal binding window in autism spectrum disorders" Experimental Brain Research 203 381-389

Freiherr J, Lundström J N, Habel U, Reetz K, 2013 “Multisensory integration mechanisms during aging" Frontiers in human neuroscience 7863

Frith C D, 2007 “The social brain?” Philosophical Transactions of the Royal Society B: Biological Sciences 362 671-678

Green D M, Dai H, 1991 "Probability of being correct with 1 of m orthogonal signals." Perception \& Psychophysics

Green D M, Swets J A, et al., 1966 Signal detection theory and psychophysics volume 1 (Wiley New York)

Heed T, Habets B, Sebanz N, Knoblich G, 2010 "Others' actions reduce crossmodal integration in peripersonal space" Current Biology 20 1345-1349

Helbig H B, Ernst M O, 2008 "Visual-haptic cue weighting is independent of modality-specific attention" Journal of Vision 8 21-21

Iani C, Anelli F, Nicoletti R, Arcuri L, Rubichi S, 2011 "The role of group membership on the modulation of joint action" Experimental Brain Research 211439

Karlinsky A, Lam M Y, Chua R, Hodges N J, 2017 "Whose turn is it anyway? the moderating role of response-execution certainty on the joint simon effect" Psychological research 1-9

Kherad-Pajouh S, Renaud O, 2015 "A general permutation approach for analyzing repeated measures anova and mixedmodel designs” Statistical Papers 56 947-967

Knoblich G, Sebanz N, 2006 "The social nature of perception and action" Current Directions in Psychological Science 15 99-104

Körding K P, Beierholm U, Ma W J, Quartz S, Tenenbaum J B, Shams L, 2007 "Causal inference in multisensory perception" PLOS ONE 2 e943

Laurienti P J, Burdette J H, Maldjian J A, Wallace M T, 2006 "Enhanced multisensory integration in older adults" Neurobiology of Aging 27 1155-1163

McClung J S, Jentzsch I, Reicher S D, 2013 “Group membership affects spontaneous mental representation: failure to represent the out-group in a joint action task" PloS one 8 e79178

McGovern D P, Roudaia E, Stapleton J, McGinnity T M, Newell F N, 2014 "The sound-induced flash illusion reveals dissociable age-related effects in multisensory integration" Frontiers in Aging Neuroscience 6250

Mishra J, Martinez A, Hillyard S A, 2008 "Cortical processes underlying sound-induced flash fusion" Brain Research 1242 102-115

Mishra J, Martínez A, Hillyard S A, 2010 "Effect of attention on early cortical processes associated with the sound-induced extra flash illusion" Journal of cognitive neuroscience 22 1714-1729

Mishra J, Martinez A, Sejnowski T J, Hillyard S A, 2007 "Early cross-modal interactions in auditory and visual cortex underlie a sound-induced visual illusion" Journal of Neuroscience $\mathbf{2 7}$ $4120-4131$

Morein-Zamir S, Soto-Faraco S, Kingstone A, 2003 “Auditory capture of vision: examining temporal ventriloquism" Cognitive Brain Research 17 154-163
Müller B C, Kühn S, van Baaren R B, Dotsch R, Brass M, Dijksterhuis A, 2011 "Perspective taking eliminates differences in co-representation of out-group members? actions" Experimental Brain Research 211 423-428

O'Keefe D J, 2007 "Brief report: post hoc power, observed power, a priori power, retrospective power, prospective power, achieved power: sorting out appropriate uses of statistical power analyses" Communication Methods and Measures 1 291-299

Parker J L, Robinson C W, 2018 "Changes in multisensory integration across the life span." Psychology and Aging 33545

Parkinson C, Wheatley T, 2015 "The repurposed social brain" Trends in Cognitive Sciences 19 133-141

R Core Team, 2017 R: A Language and Environment for Statistical Computing R Foundation for Statistical Computing Vienna, Austria

URL https: / /www.R-project.org/

Rohe T, Noppeney U, 2015 "Sensory reliability shapes perceptual inference via two mechanisms" Journal of vision 15 22-22

Santangelo V, Macaluso E, 2012 "19 spatial attention and audiovisual processing" The New Handbook of Multisensory Processes

Santangelo V, Spence C, 2008 "Crossmodal attentional capture in an unspeeded simultaneity judgement task" Visual Cognition $16155-165$

Santangelo V, Spence C, 2009 "Crossmodal exogenous orienting improves the accuracy of temporal order judgments" Experimental Brain Research 194 577-586

Saunders D R, Melcher D, van Zoest W, 2018 "No evidence of task co-representation in a joint stroop task" Psychological research $1-11$

Sebanz N, Bekkering H, Knoblich G, 2006 "Joint action: bodies and minds moving together" Trends in Cognitive Sciences $\mathbf{1 0}$ 70-76

Sebanz N, Knoblich G, Prinz W, 2003 "Representing others' actions: just like one's own?" Cognition 88 B11-B21

Sellaro R, Treccani B, Cubelli R, 2018 "When task sharing reduces interference: evidence for division-of-labour in stroop-like tasks" Psychological Research 1-16

Shams L, Iwaki S, Chawla A, Bhattacharya J, 2005a "Early modulation of visual cortex by sound: an meg study" Neuroscience letters 378 76-81

Shams L, Kamitani Y, Shimojo S, 2002 "Visual illusion induced by sound" Cognitive Brain Research 14 147-152

Shams L, Kim R, 2010 "Crossmodal influences on visual perception" Physics of Life Reviews 7 269-284

Shams L, Ma W J, Beierholm U, 2005b "Sound-induced flash illusion as an optimal percept" Neuroreport 16 1923-1927

Soto-Faraco S, Lyons J, Gazzaniga M, Spence C, Kingstone A, 2002 "The ventriloquist in motion: Illusory capture of dynamic information across sensory modalities" Cognitive brain research 14 139-146

Spence C, Pavani F, Driver J, 2004 "Spatial constraints on visualtactile cross-modal distractor congruency effects" Cognitive, Affective, \& Behavioral Neuroscience 4 148-169

Stevenson R A, Siemann J K, Woynaroski T G, Schneider B C, Eberly H E, Camarata S M, Wallace M T, 2014 "Evidence for diminished multisensory integration in autism spectrum disorders" Journal of Autism and Developmental Disorders 44 
3161-3167

Vanes L D, White T P, Wigton R L, Joyce D, Collier T, Shergill S S, 2016 "Reduced susceptibility to the sound-induced flash fusion illusion in schizophrenia" Psychiatry research 245 58-65

Vesper C, Abramova E, Btepage J, Ciardo F, Crossey B, Effenberg A, Hristova D, Karlinsky A, McEllin L, Nijssen S, Schmitz L, Wahn B, 2017 "Joint action: Mental representations, shared information and general mechanisms for coordinating with others" Frontiers in Psychology 72039

Vesper C, Butterfill S, Knoblich G, Sebanz N, 2010 “A minimal architecture for joint action" Neural Networks 23 998-1003

Vroomen J, Bertelson P, De Gelder B, 2001 "The ventriloquist effect does not depend on the direction of automatic visual attention" Perception \& Psychophysics 63 651-659

Wahn B, Dosso J A, Kingstone A, in press “Audiovisual integration during joint action: No effects for motion discrimination and temporal order judgment tasks" PsyArXiv

Wahn B, Keshava A, Sinnett S, Kingstone A, König P, 2017a "Audiovisual integration is affected by performing a task jointly" in "Proceedings of the 39th Annual Conference of the Cognitive Science Society",

Wahn B, König P, 2015a "Audition and vision share spatial attentional resources, yet attentional load does not disrupt audiovisual integration" Frontiers in Psychology 6

Wahn B, König P, 2015b "Vision and haptics share spatial attentional resources and visuotactile integration is not affected by high attentional load" Multisensory Research 28 371-392

Wahn B, König P, 2016 "Attentional resource allocation in visuotactile processing depends on the task, but optimal visuotactile integration does not depend on attentional resources" Frontiers in Integrative Neuroscience 10

Wahn B, Murali S, Sinnett S, König P, 2017b "Auditory stimulus detection partially depends on visuospatial attentional resources" $i$-Perception 82041669516688026

Williams L E, Light G A, Braff D L, Ramachandran V S, 2010 "Reduced multisensory integration in patients with schizophrenia on a target detection task" Neuropsychologia 48 3128-3136

Yamaguchi M, Clarke E L, Egan D L, 2018 "Is your color my color? dividing the labor of the stroop task between co-actors" Frontiers in psychology 9 